\title{
Clinical effect of unilateral biportal endoscopy in the treatment of lumbar diseases: a systematic review and meta-analysis
}

\author{
Xuehu Xie, Guoqiang Zhang, Ning Liu \\ Department of Orthopedics, Beijing Friendship Hospital, Capital Medical University, Beijing, China
}

Videosurgery Miniinv 2022; 17 (1): 61-68

DOI: https://doi.org/10.5114/wiitm.2021.110413

\begin{abstract}
Introduction: Currently, unilateral biportal endoscopy (UBE) as a new minimally invasive technique has been applied to conventional arthroscopic systems for the treatment of spinal disease.

Aim: To analyze the clinical effect of UBE in the treatment of lumbar diseases.

Material and methods: A systematic review of the literature published up to May 2021 was performed in the English database PubMed, Embase, and the Chinese database CNKI, Wanfang. There were 9 studies included in this systematic review and meta-analysis. The outcomes measured included operative time, Visual Analog Scale (VAS), and the Oswestry Disability Index (ODI).

Results: A total of 528 patients were collected from the selected 9 articles. The random effects model showed that the value of pooled effect mean deviation (MD) in each study was 58.62 (95\% Cl (57.53, 59.72), $p<0.001)$. At the end of postoperative follow-up, the standard mean difference (SMD) VAS scores of legs $-4.12(95 \% \mathrm{Cl}(-5.15,-3.09)$, $p<0.001)$ and back -3.10 (95\% Cl (-4.35, -1.84), $p<0.001)$ were lower than the preoperative values. At the same time, the results of the random-effects model showed that the SMD of the ODI score was -7.07 (95\% Cl (-8.69, -5.46), $p<0.001$ ) and at the end of follow-up was lower than preoperatively.

Conclusions: UBE surgery has a good clinical effect in the treatment of lumbar diseases, and can be widely used in the treatment of free prolapse lumbar disc herniation (LDH).
\end{abstract}

Key words: unilateral biportal endoscopy, lumbar disease, lumbar disc herniation, lumbar spinal stenosis.

\section{Introduction}

Lumbar discectomy and decompression are the most commonly used and classic surgical methods for treatment of lumbar diseases. They have the advantages of thorough decompression and clear vision but are associated with many complications, such as chronic low back pain [1] and spinal instability [2]. Open lumbar microdiscectomy (OLM) results in increased risks of postoperative spinal instability and chronic back pain in lumbar disc herniation [3]. Daisheng et al. studied UBE surgery for lumbar disc herniation and lumbar spinal ste- nosis, and obtained high patient satisfaction [4]. Unilateral biportal endoscopic (UBE) surgery has been used more and more to treat lumbar disc herniation or lumbar spinal stenosis (LSS), and has achieved good results [5]. In 2017, Heo et al. first proposed the concept of UBE spine technique and successfully applied it in lumbar interbody fusion surgery [6]. Similar to traditional lumbar interlaminar fenestration, the UBE establishes percutaneous access through two small cuts on each side. Operative tools are placed in the operation channel for various operations inside and outside the spinal canal [7]. A recent study of Eum et al. reported

\section{Address for correspondence}

Guoqiang Zhang, Department of Orthopedics, Beijing Friendship Hospital, Capital Medical University, Beijing, China,

e-mail: zgq20102012@163.com 
the favorable outcomes of UBE after development of HD endoscopic vision, showing that the ODI score decreased from $67.2 \pm 1.7$ to $24.3 \pm 8.5$, and the VAS for leg pain decreased from $8.3 \pm 1.1$ to 2.4 $\pm 1.1[8]$.

Compared with conventional open lumbar microdiscectomy (OLM), UBE has advantages of less intraoperative blood loss and postoperative back pain and relatively shorter hospital stay due to the preservation of the back muscle and a smaller incision. These advantages extend the scope of lumbar spinal stenosis, degenerative diseases of the cervical spine, and even short-level fusion procedures [9]. However, Korean researchers compared the results of the UBE and OLM procedures, and found that patients who underwent UBE surgery had less intraoperative blood loss and greater improvement in short-term back pain, while having similar short-term leg pain, long-term lumbago and leg pain, DOI, satisfaction, and complication rates to patients who underwent OLM [10].

\section{Aim}

To better understand the application of UBE surgery in the treatment of degenerative diseases of the lumbar spine. Therefore a meta-analysis was used to measure the effect of UBE on patients after surgery.

\section{Material and methods}

\section{Search of literature}

We performed this meta-analysis following PRISMA guidelines. All the prospective or retrospective studies published from the database inception until May 2021 were searched from two English-language databases (PubMed and Embase) and two Chinese-language databases (China National Knowledge Infrastructure and Wanfang) by 2 reviewers. Search terms were ((unilateral biportal endoscopy OR UBE) AND (lumbar disc herniation OR LDH OR lumbar spinal stenosis OR LSS OR degenerative lumbar spinal stenosis OR DLSS)).

\section{Inclusion and exclusion criteria}

The inclusion criteria included (1) study population: patients with lumbar disc herniation or lumbar disc stenosis (2) study design: prospective/retrospective cohort studies; (3) operation method: uni- lateral biportal endoscopy; (4) indicators of clinical outcomes: operative time, Visual Analog Scale (VAS) and Oswestry Disability Index (ODI).

The exclusion criteria were (1) duplicated article; (2) conference summaries, comments, letters, etc.; (3) animal studies, existing meta-analyses and systematic reviews; (4) studies on other surgical methods of treatment.

\section{Data extraction}

Data collection proceeded in terms of first author name, publication years, study type, number of patients, study period, operation time, diagnosis, and follow-up period. The collected outcomes were operation time, VAS scores for back and leg, and ODI. Data were extracted from the literature by the first reviewer, and accuracy was confirmed by the second reviewer.

The Newcastle-Ottawa Scale adapted for nonrandomized controlled studies was applied to evaluate the quality of the studies. This scale has three sections: 1 - selection (3 items, maximum score: 3 points), 2 - comparability (1 item, maximum score: 2 points), and 3 - outcome ( 2 items, maximum score: 3 points). The studies were then assigned to one of the following categories accordingly: very good studies: 7-8 scores; good studies: 5-6 scores; satisfactory studies: $3-2$ scores; unsatisfactory studies: 0-1 score.

\section{Statistical analysis}

Data analysis was performed using Stata software. Standard mean difference (SMD) or mean deviation (MD) and $95 \% \mathrm{Cl}$ were used to evaluate the overall effect of the continuous index. In a sensitivity analysis, we sequentially removed one study at a time and re-analyzed the data to determine whether any one study was influencing the results. There was no bias analysis or subgroup analysis due to the small number of literature references included in this study. Based on the heterogeneity test results, random effects or fixed effects models were selected to estimate the total effects. The $\mathrm{Q}$ test and $l^{2}$ test were used to estimate inter-study heterogeneity. When $p>0.1$ and $l^{2} \leq 50 \%$, the fixed effects model was adopted. When $p>0.1$ and $l^{2} \geq$ $50 \%$, the random effects model was used. $P<0.05$ was considered statistically significant, whereas $p>$ 0.05 was considered not significant. 


\section{Results}

\section{Study selection}

A total of 73 studies were identified through the database search. Twenty-one duplicate reports were excluded. After title and abstract screening that excluded 16 irrelevant studies, 16 reports were excluded due to irrelevant topics. According to the inclusion and exclusion criteria, 27 studies were excluded, while 9 studies met the inclusion criteria. A specific study flowchart is shown in Figure 1 and the selected study characteristics are listed in Table I. The quality score of all included studies was $>6$, indicating good quality.

\section{Operation time of UBE}

The results of the random effects model showed that the pooled effect value of the operation time for the treatment of lumbar diseases with UBE was 58.62 ( $95 \% \mathrm{Cl}(57.53,59.72), p<0.001)$. Heterogeneity exists among studies $\left(I^{2}=94.1 \%, p<0.001\right.$, Figure 2).

\section{Comparison of VAS and ODI scores before and after surgery}

The results of the random-effect model showed that the VAS score of legs at the end of the follow-up was lower than that before surgery. At the end of postoperative follow-up, the standard mean difference (SMD) VAS score of the legs was $-4.12(95 \% \mathrm{Cl}$ $(-5.15,-3.09), p<0.001)$. Heterogeneity exists among studies $\left({ }^{2}=94.7 \%, p<0.001\right.$, Figure 3$)$. Af-

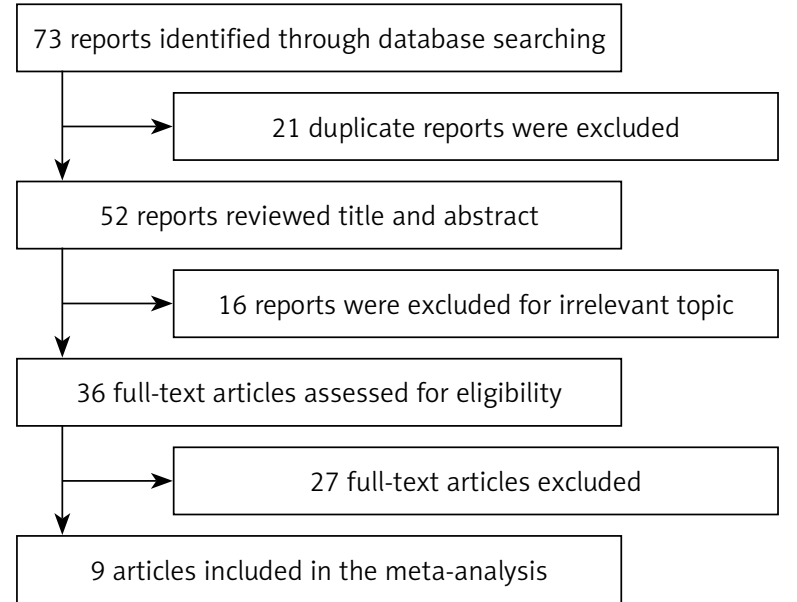

Figure 1. The process of selecting articles for the meta-analysis

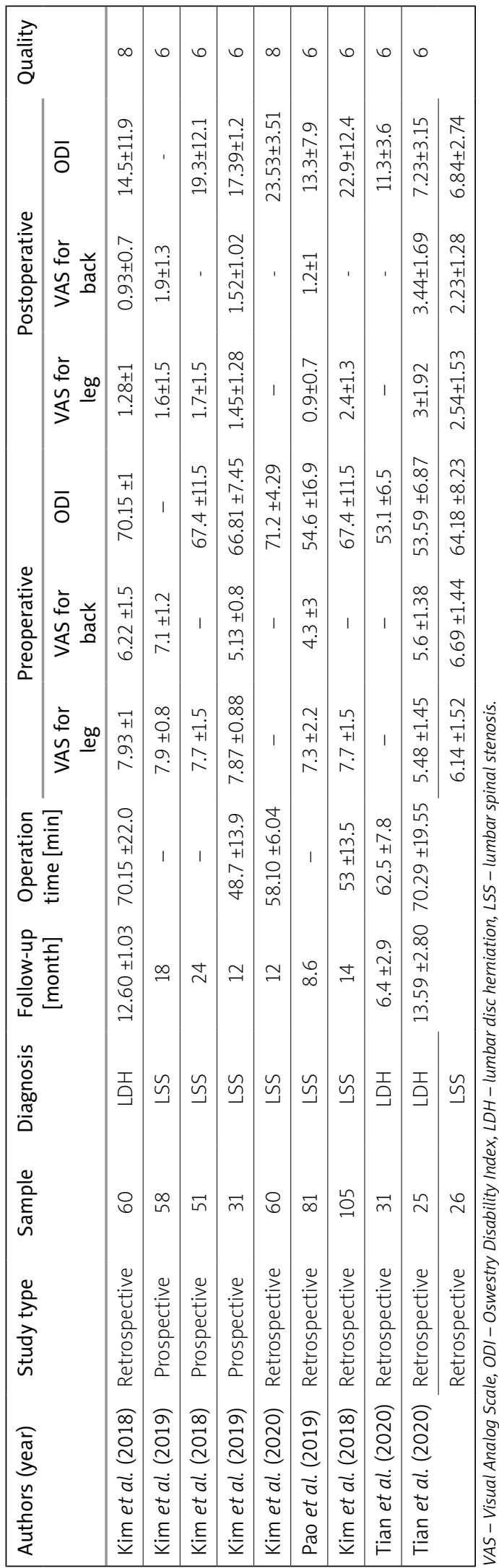


Study ID Operative time

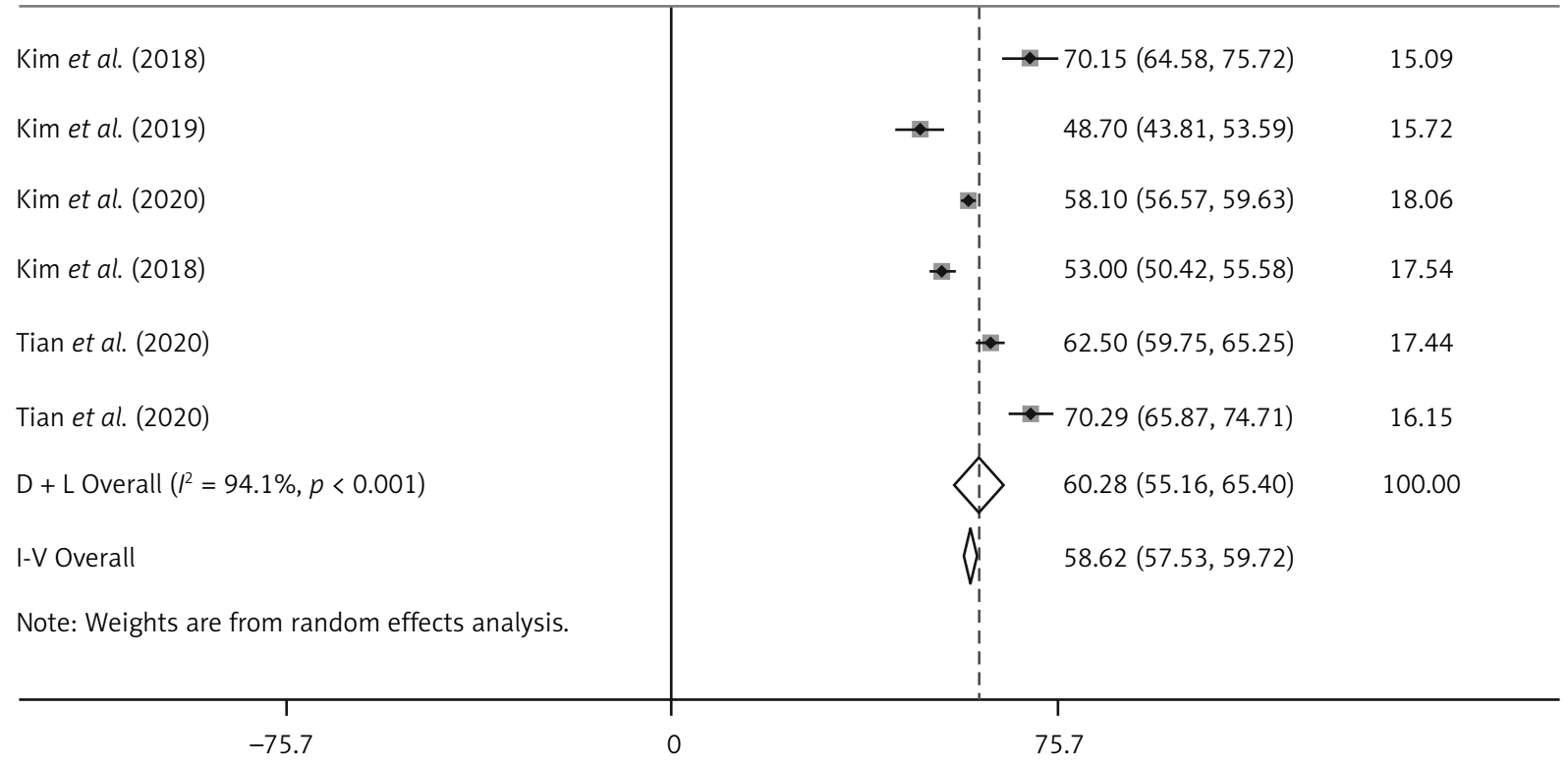

Figure 2. The combined effect results of operation time in each study

\begin{tabular}{cc} 
SMD $(95 \% \mathrm{Cl})$ & Weight (\%) \\
\hline$-6.65(-7.57,-5.73)$ & 12.10 \\
$-5.24(-6.01,-4.47)$ & 12.46 \\
$-4.00(-4.68,-3.32)$ & 12.66 \\
$-5.85(-7.00,-4.69)$ & 11.45 \\
$-3.92(-4.45,-3.39)$ & 12.94 \\
$-3.78(-4.23,-3.32)$ & 13.05 \\
$-1.46(-2.08,-0.83)$ & 12.76 \\
$-2.36(-3.07,-1.65)$ & 12.58 \\
$-4.12(-5.15,-3.09)$ & 100.00
\end{tabular}

Overall $\left(I^{2}=94.7 \%, p<0.001\right)$

NOTE: Weights are from random effects analysis!

$$
-7.57
$$

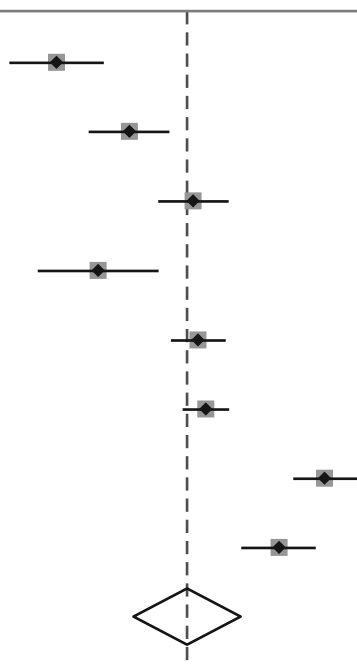

$-4.12(-5.15,-3.09)$

Figure 3. Comparison of combined effects of VAS for leg scores before and after surgery in different studies

ter each study was removed successively, the pooled score of the legs was $-3.10(95 \% \mathrm{Cl}(-4.35,-1.84)$, effect values were all in the range of 95\% $(-5.15, \quad p<0.001)$. Heterogeneity exists among studies $\left(I^{2}=\right.$ -3.09), and the study results were stable (Figure 4$) . \quad 96.0 \%, p<0.001$, Figure 5 ). After each study was reThe results of the random-effect model showed that the VAS score of the back at the end of follow-up after surgery was lower than that before surgery. At the end of postoperative follow-up, the SMD VAS moved successively, the pooled effect values were all in the range of $95 \% \mathrm{Cl}(-4.35,-1.84)$, and the study results were stable (Figure 6 ). The results of the random-effects model showed that the SMD of the ODI 
score was $-7.07(95 \% \mathrm{Cl}(-8.69,-5.46), p<0.001)$ and at the end of follow-up was lower than the preoperative value. Heterogeneity exists among studies $\left(I^{2}=96.8 \%, p<0.001\right.$, Figure 7$)$. After each study was removed successively, the pooled effect values were all in the range of $95 \% \mathrm{Cl}(-8.69,-5.46), p<0.001)$, and the study results were stable (Figure 8 ).

\section{Discussion}

Although conventional open laminotomy and discectomy provide an effective way for symptomatic herniation treatment, muscle and ligament injury from surgery can lead to postoperative back pain and muscle atrophy. Therefore, after traditional surgery, patients need more time for functional recovery and pain control. Postoperative back pain following mechanical trauma due to OLM has already been reported. Dvorak et al. reported that $70 \%$ of patients experienced back pain after conventional discectomy during long-term follow-up [11]. Parker et al. also reported that $32 \%$ of patients suffered back pain after conventional discectomy, and $9 \%$ of cases underwent fusion surgery for pain control [12]. Vodicar et al. reported that invasive procedures, including endplate perforation, decrease vertebral height and worsen back pain in the postoperative period [13].

In recent years, with the development of endoscopic techniques and surgical instruments, UBE

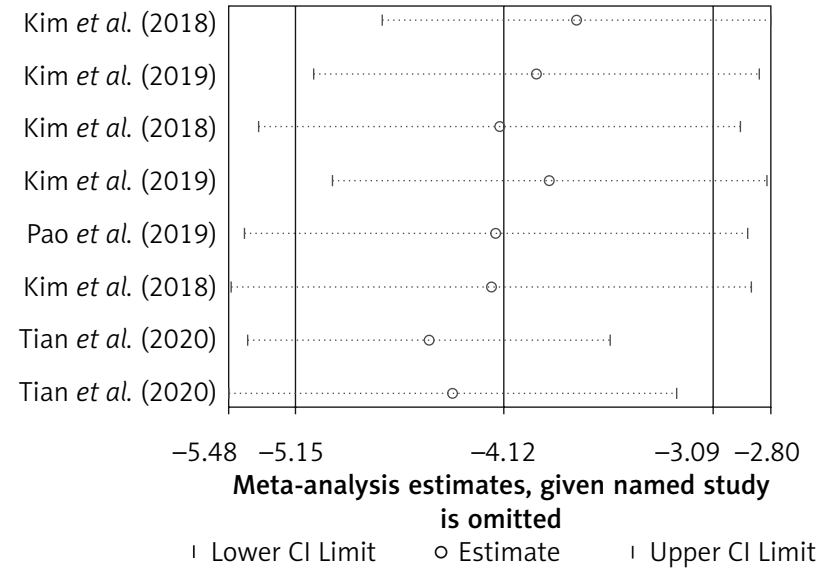

Figure 4. Sensitivity analysis of VAS for leg scores

technique has been successfully applied to a variety of diseases involving cervical, thoracic and lumbar vertebrae [14-16]. UBE can achieve high-resolution visualization at a small muscle dissection and use almost all laminectomy instruments without restriction. HD endoscopic vision makes disc dissection easier and ruptured fragment removal and manipulation is possible as in the conventional technique. Because the same instruments are used while allowing for a more detailed view than in microscopic surgery, favorable radiologic outcomes can be achieved.

UBE is a new method that combines the advantages of interlaminar endoscopy and microscopic

\section{Study ID VAS for back}

SMD $(95 \% \mathrm{Cl}) \quad$ Weight $(\%)$

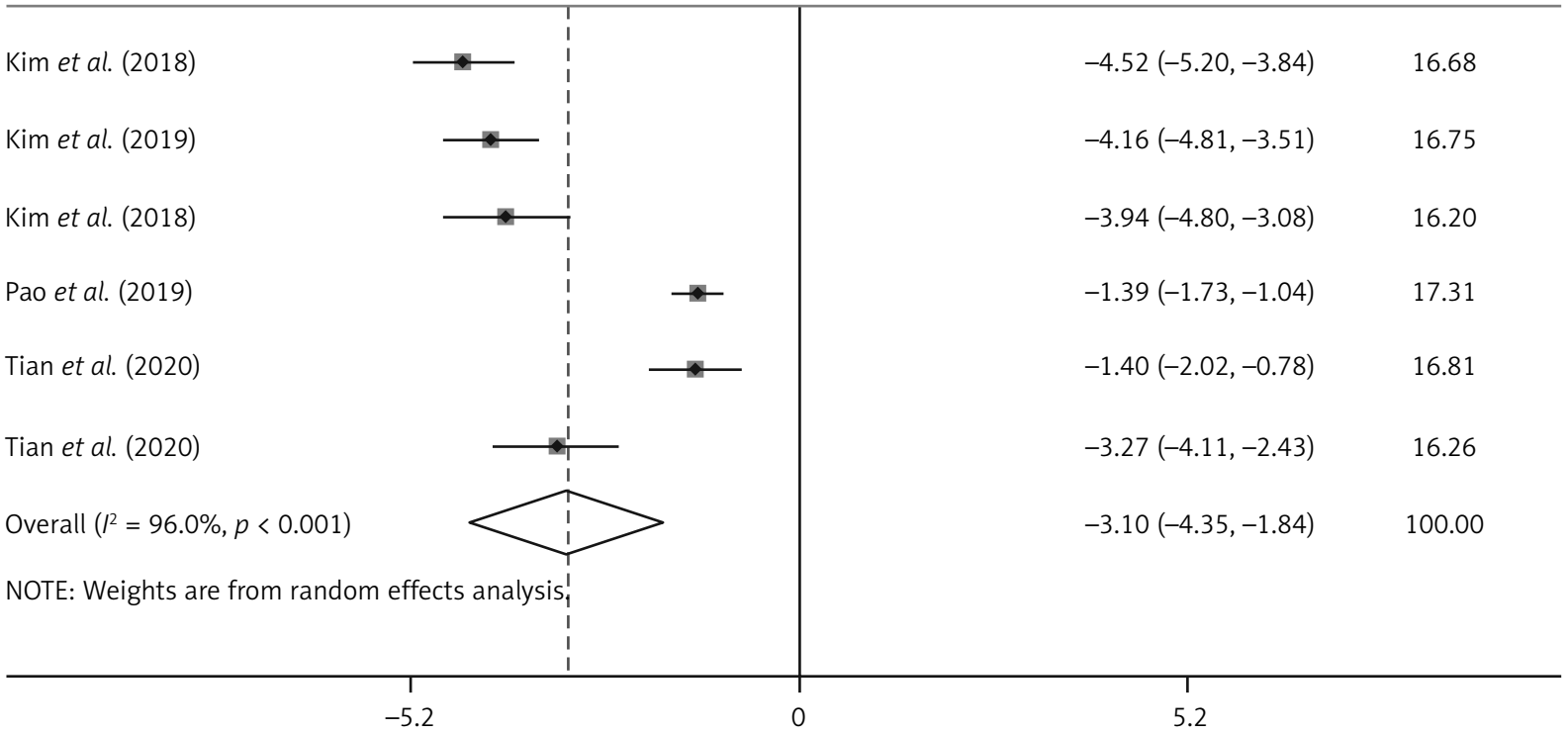

Figure 5. Comparison of combined effects of VAS for back scores before and after surgery in different studies 


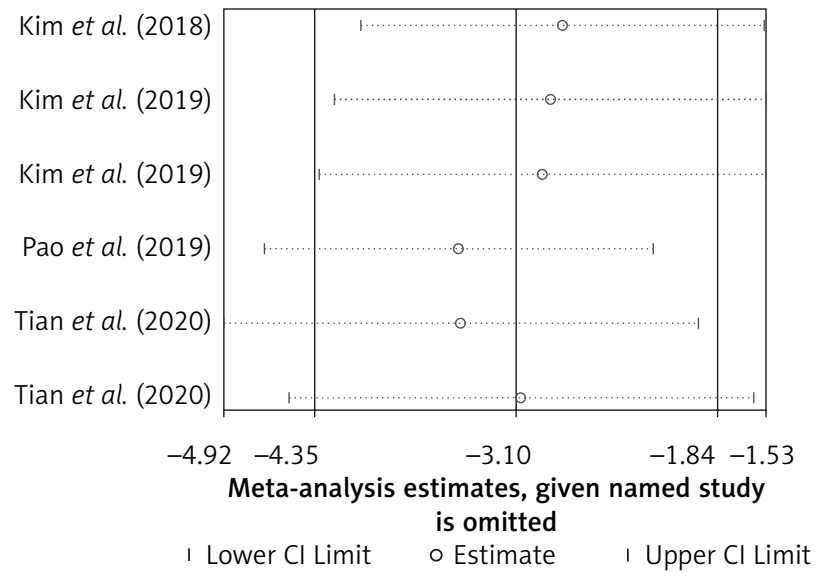

Figure 6. Sensitivity analysis of VAS for back scores

surgery. UBE surgery is more and more widely used in clinical treatment. Kambin et al. reported a high rate of $87 \%$ patient satisfaction for arthroscopic disc surgery [17]. Casey et al. assessed the radiologic outcomes of arthroscopic discectomy and found that the success rates based on CT and MRI were $88.9 \%(n=18)$ and $85.7 \%(n=12)$, respectively [18]. Kim et al. reported that pre-operative VAS back was $6.22 \pm 1.5$, but post-operative VAS back was $0.93 \pm 0.7$ [10]. Pao et al. found that the patients had the most significant improvement in VAS score for leg pain, deceasing from 7.3 to only 0.9 , as well as significant improvement in neurological symptoms and disability status, reflected in the improvement of JOA scores and ODI [19]. In Lin et al.'s systematic review the results showed that the VAS score and ODI score of the nine studies included were improved compared with the preoperative score at the final follow-up, indicating that the postoperative function of the patients was greatly improved [20]. Consistent with this conclusion, in our study, VAS scores of both legs and back were significantly decreased after UBE surgery compared with those before surgery. The results of the random effects model showed that the ODI score at the end of follow-up was significantly lower than that before the operation. The combined effect value was stable.

The duration of UBE surgery is longer than that of OLM, so patients experience longer periods of pain. The time is long because UBE can provide a clearer approach to the affected site, avoid damage to spinal stability caused by surgical procedures, and finally achieve the desired clinical results. Therefore more clinical studies are needed to prove the advantages of UBE surgery. The clinical symptoms of 31 patients were significantly improved after the operation. ODI and VAS scores were significantly lower than those before surgery, and continued to decline over time [21]. UBE surgery causes less damage to the stable

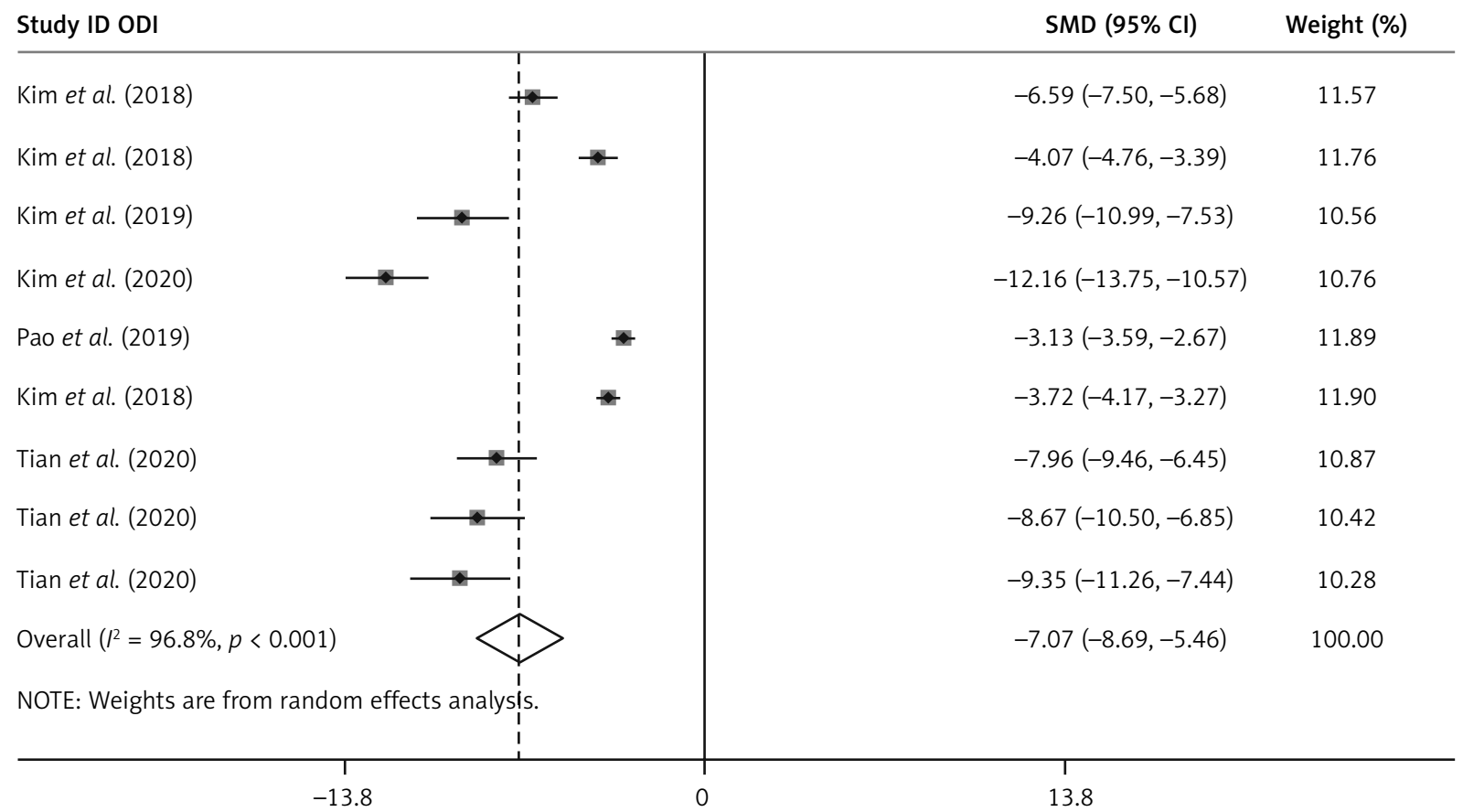

Figure 7. Comparison of combined effects of ODI scores before and after surgery in different studies 


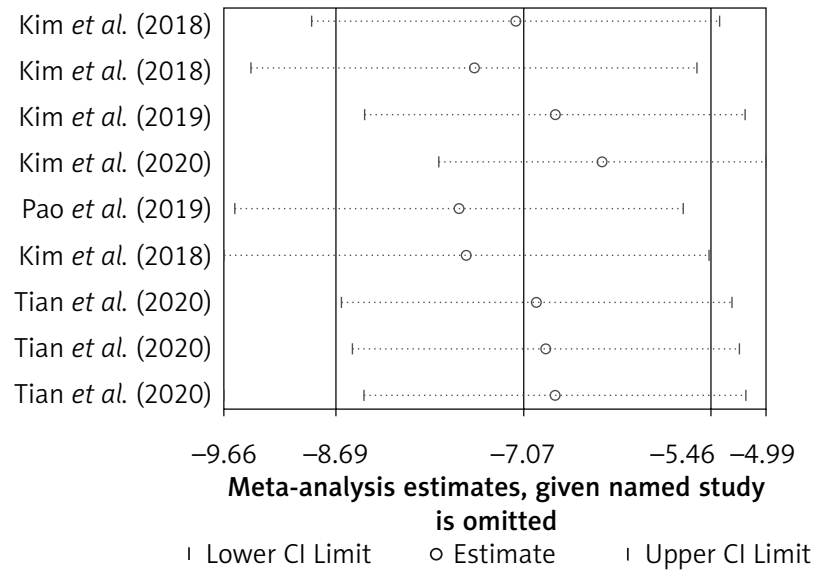

Figure 8 . Sensitivity analysis of ODI

structures of the spine, such as the facet structures. Postoperative CT showed that the articular process and contralateral lamina were intact. Postoperative recovery is fast; the next day the patient can get out of bed. This method should be accepted and widely used in more diseases.

\section{Conclusions}

The UBE technique has a satisfactory effect in the treatment of lumbar intervertebral disc disease and is worth popularizing. It provides a new direction for the treatment of lumbar disc diseases. The number of randomized controlled trials and prospective studies included was too small. Sufficient randomized prospective studies of UBE are needed to validate the current results. There are some differences in the surgical techniques used by each doctor. Due to the small number of studies, no subgroup analysis was performed.

\section{Conflict of interest}

The authors declare no conflict of interest.

\section{References}

1. Hu ZJ, Fang XQ, Zhou ZJ, et al. Effect and possible mechanism of muscle-splitting approach on multifidus muscle injury and atrophy after posterior lumbar spine surgery. J Bone Joint Surg Am 2013; 95: e192(1-9).

2. Hartmann F, Janssen C, Böhm S, et al. Biomechanical effect of graded minimal-invasive decompression procedures on lumbar spinal stability. Arch Orthopaedic Trauma Surg 2012; 132: 1233-9.

3. Carragee EJ, Han MY, Suen PW, et al. Clinical outcomes after lumbar discectomy for sciatica: the effects of fragment type and anular competence. J Bone Joint Surg Am 2003; 85: 102-8.
4. Daisheng T, Bin Z, Jianjun L, et al. Unilateral double channel endoscopy for the treatment of free prolapse lumbar disc herniation. Chin I Minimally Invasive Surg 2020; 20: 33-7.

5. Eun SS, Eum JH, Lee SH, et al. Biportal endoscopic lumbar decompression for lumbar disk herniation and spinal canal stenosis: a technical note. J Neurol Surg A Cent Eur Neurosurg 2017; 78: 390-96.

6. Heo DH, Son SK, Eum JH, et al. Fully endoscopic lumbar interbody fusion using a percutaneous unilateral biportal endoscopic technique: technical note and preliminary clinical results. Neurosurg Focus 2017; 43: E8.

7. Pranata R, Lim MA, Vania R, et al. Biportal endoscopic spinal surgery versus microscopic decompression for lumbar spinal stenosis: a systematic review and meta-analysis. World Neurosurg 2020; 138: e450-58.

8. Hwa Eum J, Hwa Heo D, Son SK, et al. Percutaneous biportal endoscopic decompression for lumbar spinal stenosis: a technical note and preliminary clinical results. J Neurosurg Spine 2016; 24: 602-7.

9. Mayer HM, Brock M, Berlien HP, et al. Percutaneous endoscopic laser discectomy (PELD). A new surgical technique for non-sequestrated lumbar discs. Acta Neurochirur Suppl 1992; 54: 53-8.

10. Kim SK, Kang SS, Hong YH, et al. Clinical comparison of unilateral biportal endoscopic technique versus open microdiscectomy for single-level lumbar discectomy: a multicenter, retrospective analysis. J Orthop Surg Res 2018; 13: 22.

11. Dvorak J, Gauchat MH, Valach L. The outcome of surgery for lumbar disc herniation. I. A 4-17 years' follow-up with emphasis on somatic aspects. Spine 1988; 13: 1418-22.

12. Parker SL, Xu R, McGirt MJ, et al. Long-term back pain after a single-level discectomy for radiculopathy: incidence and health care cost analysis. J Neurosurg Spine 2010; 12: 178-82.

13. Vodičar $M$, Košak R, Gorenšek $M$, et al. Vertebral end-plate perforation for intervertebral disc height preservation after single-level lumbar discectomy: a randomized-controlled trial. Clin Spine Surg 2017; 30: E707-e12.

14. Akbary K, Kim JS, Park CW, et al. Biportal endoscopic decompression of exiting and traversing nerve roots through a single interlaminar window using a contralateral approach: technical feasibilities and morphometric changes of the lumbar canal and foramen. World Neurosurg 2018; 117: 153-61.

15. Kim JE, Choi DJ. Bi-portal Arthroscopic Spinal Surgery (BASS) with $30^{\circ}$ arthroscopy for far lateral approach of L5-S1 - technical note. J Orthop 2018; 15: 354-58.

16. Osman SG, Schwartz JA, Marsolais EB. Arthroscopic discectomy and interbody fusion of the thoracic spine: areport of ipsilateral 2-portal approach. Int I Spine Surg 2012; 6: 103-9.

17. Kambin P, O’Brien E, Zhou L, et al. Arthroscopic microdiscectomy and selective fragmentectomy. Clin Orthop Relat Res 1998; 347: 150-67.

18. Casey KF, Chang MK, O'Brien ED, et al. Arthroscopic microdiscectomy: comparison of preoperative and postoperative imaging studies. Arthroscopy 1997; 13: 438-45.

19. Pao JL, Lin SM, Chen WC, et al. Unilateral biportal endoscopic decompression for degenerative lumbar canal stenosis. J Spine Surg 2020; 6: 438-46. 
20. Lin GX, Huang P, Kotheeranurak V, et al. A systematic review of unilateral biportal endoscopic spinal surgery: preliminary clinical results and complications. World Neurosurg 2019; 125: 425-32.

21. Tian D, Zhu B, Liu J, et al. Unilateral double channel endoscopy for the treatment of free prolapse lumbar disc herniation. Chin J Minim Invasive Surg 2020; 20: 33-7.

Received: 2.08.2021, accepted: 19.09.2021 